\title{
Efficacy of Pembrolizumab Monotherapy for Advanced Gastric/Gastroesophageal Junction Cancer with Programmed Death Ligand 1 Combined Positive Score $\geq 10$ Ac
}

Zev A. Wainberg' ${ }^{1}$ Charles S. Fuchs ${ }^{2}$, Josep Tabernero ${ }^{3}$, Kohei Shitara ${ }^{4}$, Kei Muro ${ }^{5}$, Eric Van Cutsem ${ }^{6}$, Yung-Jue Bang ${ }^{7}$, Hyun Cheol Chung ${ }^{8}$, Kensei Yamaguchi ${ }^{9}$, Eniko Varga ${ }^{10}$, Jen-Shi Chen", Daniel Hochhauser ${ }^{12}$, Peter Thuss-Patience ${ }^{13}$, Salah-Eddin Al-Batran ${ }^{14}$, Marcelo Garrido ${ }^{15}$, Uma Kher ${ }^{16}$, Chie-Schin Shih ${ }^{16}$, Sukrut Shah ${ }^{16}$, Pooja Bhagia ${ }^{16}$, and Joseph Chao ${ }^{17}$

\section{ABSTRACT}

Purpose: Pembrolizumab demonstrated efficacy in PD-L1-positive [combined positive score (CPS) $\geq 1$ ] advanced gastric/gastroesophageal junction (G/GEJ) cancer in the first-, second-, and thirdline setting in KEYNOTE-062, KEYNOTE-061, and KEYNOTE059, respectively. To better delineate the specificity of CPS as a predictor of clinical outcomes, we analyzed pembrolizumab efficacy in patients with CPS $\geq 10$ in these trials.

Patients and Methods: Included were patients with CPS $\geq 10$ tumors from KEYNOTE-059 cohort 1 (pembrolizumab, $n=46$; post hoc), KEYNOTE-061 (pembrolizumab, $n=53$; chemotherapy, $n=55$; post hoc), and KEYNOTE-062 (pembrolizumab, $n=92$; chemotherapy, $n=90$; primary). Efficacy outcomes were overall survival (OS), progression-free survival (PFS), objective response rate (ORR), and duration of response (DOR).

Results: In KEYNOTE-059, median follow-up was 6 months, median OS was 8 months [95\% confidence interval (CI), 5.8-11.1],

\section{Introduction}

Gastric cancer ranks fifth among the most commonly diagnosed cancers worldwide and accounts for more than 1 million new cases and approximately 800,000 deaths per year (1). Evidence suggests that the programmed death 1 (PD-1) pathway may have prognostic significance in gastric cancer, with several studies demonstrating a relationship between expression of PD-L1 and overall survival (OS; refs. 2-4). Although the prevalence of immunohistochemical PD-L1 expression varies between studies, most indicate that a significant proportion (range, 25\%-65\%) of patients with gastric cancer overexpress PD-L1,

'Department of the Division of Hematology Oncology, David Geffen School of Medicine at UCLA, Los Angeles, California. ${ }^{2}$ Department of Medical Oncology, Yale Cancer Center, Smilow Cancer Hospital, New Haven, Connecticut. ${ }^{3}$ Department of Medical Oncology, Vall d'Hebron Hospital Campus and Institute of Oncology (VHIO), IOB-Quiron, UVic-UCC, Barcelona, Spain. ${ }^{4}$ Department of Gastrointestinal Oncology, National Cancer Center Hospital East, Chiba, Japan. ${ }^{5}$ Department of Clinical Oncology, Aichi Cancer Center Hospital, Nagoya, Japan. ${ }^{6}$ University Hospitals and KU Leuven, Leuven, Belgium. ${ }^{7}$ Department of Internal Medicine, Seoul National University College of Medicine, Seoul, Republic of Korea (South). ${ }^{8}$ Department of Internal Medicine, Yonsei Cancer Center, Yonsei University College of Medicine, Seoul, Republic of Korea (South). ${ }^{9}$ Department of Gastroenterological Chemotherapy, The Cancer Institute Hospital of JFCR, Tokyo, Japan. ${ }^{10}$ Department of Oncology, Debreceni Egyetem Klinikai Központ, Debrecen, Hungary. "Department of Hematology, Oncology, Linkou Chang Gung Memorial Hospital and Chang Gung University, Tao-Yuan, Taiwan. ${ }^{12}$ Department of Medical Oncology, UCL Cancer Institute, London, United Kingdom. ${ }^{13}$ Department of Hematology, Oncology, and Tumor Immunology,
ORR was $17 \%$, and median (range) DOR was 21 months (3+ to 35+). In KEYNOTE-061, median follow-up was 9 months, median OS (pembrolizumab vs. chemotherapy) was 10 versus 8 months (HR, 0.64; 95\% CI, 0.41-1.02), median PFS was 3 months versus 3 months (HR, 0.86; $95 \% \mathrm{CI}, 0.56-1.33$ ), ORR was $25 \%$ versus $9 \%$, and median (range) DOR was not reached ( 4 to $26+$ months) versus 7 months (3-7). In KEYNOTE-062, median follow-up was 11 months, median OS (pembrolizumab vs. chemotherapy) was 17 months versus 11 months (HR, 0.69 ; 95\% CI, 0.49-0.97), median PFS was 3 months versus 6 months (HR, 1.09, 95\% CI; 0.79-1.49), ORR was $25 \%$ versus $38 \%$, and median (range) DOR was 19 months $(1+$ to $34+)$ versus 7 months $(2+$ to $30+)$.

Conclusions: This comprehensive analysis showed consistent improvements toward more favorable clinical outcomes with pembrolizumab across lines of therapy in patients with CPS $\geq 10 \mathrm{G} / \mathrm{GEJ}$ cancer.

regardless of scoring method $(2,5)$. Current first-line standard-of-care therapy for patients with unresectable locally advanced recurrent or metastatic disease remains combination chemotherapy with a fluoropyrimidine and a platinum-based agent, with trastuzumab added to the regimen for patients with HER2-positive disease (6). Various agents are recommended for use in second-line therapy, including chemotherapies and immunotherapies. The anti-PD-1 inhibitor pembrolizumab is approved for the treatment of patients with gastric cancer and is among the preferred regimens as second-line therapy for patients with microsatellite instability-high (MSI-H) or mismatch

Charité-University Medicine Berlin, Campus Virchow-Klinikum, Berlin, Germany. ${ }^{14}$ Deparment of Tumor Diseases, Institut für Klinisch-Onkologische, Frankfurt, Germany. ${ }^{15}$ Department of Hematology and Medical Oncology, Pontificia Universidad Católica de Chile, Santiago, Chile. ${ }^{16}$ Department of Medical Oncology, Merck \& Co., Inc., Kenilworth, New Jersey. ${ }^{17}$ Department of Medical Oncology and Therapeutics Research, City of Hope Comprehensive Cancer Center, Duarte, California.

Corresponding Author: Zev A. Wainberg, Division of Hematology/Oncology, David Geffen School of Medicine at UCLA, 2825 Santa Monica Blvd, Suite 200, Santa Monica, CA, 90404. Phone: 310-633-8400; Fax: 310-586-0841;

E-mail: zwainberg@mednet.ucla.edu

Clin Cancer Res 2021;27:1923-31

doi: 10.1158/1078-0432.CCR-20-2980

(c)2021 American Association for Cancer Research. 


\section{Translational Relevance}

Pembrolizumab monotherapy demonstrated a clinically meaningful survival benefit and durable antitumor activity in patients with PD-L1 combined positive score (CPS) $\geq 10$ gastric or gastroesophageal junction cancer from KEYNOTE-059 cohort 1 ( $n=46$; third-line or later setting), KEYNOTE-061 ( $n=53$; second-line setting), and KEYNOTE-062 ( $n=92$; first-line setting). We observed numerically higher overall survival medians, response rates, and durations of response with pembrolizumab monotherapy than with chemotherapy in patients whose tumors expressed CPS $\geq 10$ across lines of therapy. Responsiveness to immune checkpoint inhibitors and the role of pembrolizumab in the treatment paradigm of gastric cancer are still being determined, and this study adds to the existing body of evidence that the immunohistochemical PD-L1 CPS is one clinically relevant biomarker that can lead to improved clinical efficacy.

protein repair-deficient (dMMR) gastric cancer. On the basis of results from KEYNOTE-059, pembrolizumab is also approved as third-line or later therapy for patients with tumors that have a PD-L1 combined positive score (CPS) $\geq 1(7)$. The ability of PD-L1 expression to predict response to immune checkpoint inhibitors beyond the approved use of third-line pembrolizumab for gastric cancer expressing CPS $\geq 1$ remains unclear.

Pembrolizumab has demonstrated antitumor activity in patients with PD-L1-positive advanced gastric or gastroesophageal junction (GEJ) cancer in phase II and III trials (8-10). In cohort 1 of the global, single-arm, multicohort, phase II KEYNOTE-059 study, patients with advanced gastric or GEJ cancer whose disease progressed after $\geq 2$ lines of therapy received pembrolizumab monotherapy (8). Among the 148 patients with CPS $\geq 1$ tumors that were either microsatellite stable or had unknown MMR/dMMR status, 23 patients had a response, for an objective response rate (ORR) of $15.5 \%$. The median duration of response (DOR) among these patients was 16.3 months (range, $1.6+$ to $17.3+$ ), and safety was manageable. Although the ORR was higher in patients with PD-L1-positive tumors (15.5\%) than in patients with PD-L1-negative tumors (6.4\%), the responses observed in the PD-L1-negative population indicated an incomplete separation of responders from nonresponders based on CPS $\geq 1$ (8).

In the randomized, open-label, phase III KEYNOTE-061 study, patients with advanced gastric or GEJ cancer whose disease progressed after first-line therapy received pembrolizumab or paclitaxel (9). Among the 395 patients with CPS $\geq 1$ tumors, pembrolizumab did not significantly prolong survival compared with paclitaxel [median OS, 9.1 vs. 8.3 months; HR, 0.82; 95\% confidence interval (CI), $0.66-$ 1.03; one-sided $P=0.0421]$. Although there was also no improvement in progression-free survival (PFS) or response rates, pembrolizumab monotherapy did offer more durable responses and a favorable safety profile compared with paclitaxel.

The randomized phase III KEYNOTE-062 study enrolled patients with advanced gastric or GEJ cancer who had not previously received therapy for advanced disease (10). Among the 506 patients with CPS $\geq 1$ tumors, OS with pembrolizumab was noninferior to that with cisplatin plus 5-fluorouracil (5-FU) or capecitabine (HR, 0.91; 99.2\% CI, 0.69-1.18; prespecified noninferiority margin, 1.2). Pembrolizumab did not improve PFS or ORR but demonstrated a better tolerability profile than chemotherapy.
The predictive value of PD-L1 in gastric cancer is unclear given that multiple studies with immune checkpoint inhibitors other than pembrolizumab have demonstrated similar responses in patients regardless of PD-L1 status. In addition, the absence of a standard PD-L1 IHC assay and scoring method across studies makes cross-study comparisons difficult. In the phase I/II CheckMate-032 study of patients with chemotherapy-refractory advanced esophagogastric cancer, responses were observed with nivolumab alone and with nivolumab in combination with ipilimumab regardless of PD-L1 status [defined as tumor proportion score (TPS) with a cutoff of $1 \%$ using PD-L1 IHC 28-8 pharmDx (Agilent Technologies; ref. 11]. Response rates were numerically higher in patients with PD-L1-positive tumors, but the sample sizes were small. The phase III ATTRACTION-2 study randomly assigned patients with advanced gastric or GEJ cancer who had previously received two or more lines of therapy to receive nivolumab or placebo (12). In an exploratory analysis evaluating PD-L1 expression (defined as TPS with a cutoff of $1 \%$ ) and OS, median OS was numerically higher with nivolumab than with placebo regardless of PD-L1 positivity. Outcomes based on PD-L1 status were also evaluated with avelumab in patients with gastric cancer in the phase Ib JAVELIN Solid Tumor trial (13), the phase III JAVELIN Gastric 300 trial (14), and the phase III JAVELIN Gastric 100 trial (15). There were no significant differences in outcomes among patients with PD-L1positive or PD-L1-negative tumors. For all three studies, PD-L1 positive was defined as $\geq 1 \%$ of tumor cells using PD-L1 IHC 73-10 pharmDx. However, exploratory analysis using 22C3 pharmDx suggested a survival benefit with maintenance avelumab over chemotherapy in patients with CPS $\geq 1$ tumors (HR, 0.72; 95\% CI, 0.49-1.05; refs. 15 and 16).

In addition to measuring PD-L1 expression on tumor cells and before the development of CPS, pembrolizumab studies assessed response by mononuclear inflammatory cell density score (MIDS). The CheckMate-032, ATTRACTION-2, and JAVELIN Gastric studies did not evaluate MIDS, which might have provided different results, highlighting the need to continue exploring patient subgroups likely to respond to $\mathrm{PD}-1 / \mathrm{PD}-\mathrm{L} 1$ inhibitors.

Among the limited PD-L1 data available for patients with gastric or GEJ cancer, the open-label phase Ib KEYNOTE-012 study (NCT01848834) evaluated the antitumor activity of pembrolizumab in patients with PD-L1-positive recurrent or metastatic adenocarcinoma of the stomach or GEJ (17). PD-L1 expression was measured in 35 patients with available biopsy samples at baseline using TPS and MIDS. When response was evaluated using TPS, ORR was $24 \%$ for patients with TPS $0 \%, 0 \%$ for patients with TPS $1 \%$ to $49 \%$, and $33 \%$ for patients with TPS $\geq 50 \%$. When response was evaluated using MIDS, ORR was $0 \%$ for MIDS 0, 25\% for MIDS 1, 12\% for MIDS 2, 44\% for MIDS 3, and $0 \%$ for MIDS 4 . Although conclusions are limited because of the small numbers of patients, these findings do not demonstrate an association between response and high PD-L1 expression using TPS though there may be an association between high MIDS and response. The study provided evidence of the importance of measuring PD-L1 expression in immune cells, as opposed to tumor cells exclusively, in patients with gastric cancer based on analysis of the results and on the use of CPS. In the CheckMate-649 study in patients with gastric or GEJ cancer or esophageal adenocarcinoma, nivolumab plus chemotherapy provided statistically significant improvements in OS and PFS compared with chemotherapy alone in patients with CPS $\geq 5$ tumors (18). A statistically significant OS benefit was also shown in patients with CPS $\geq 1$ tumors and in the all-randomly assigned population, showing an enrichment of OS benefit as the CPS cutoff increased (18). 
A recent meta-analysis of randomized controlled trials of PD-1/ PD-L1 inhibitors in patients with advanced solid tumors, including three trials in patients with gastric or GEJ cancer, suggested that enriching for PD-L1 status by increasing the minimum proportion of stained cells can increase efficacy in a dose-response relationship (19). On the basis of the experience with pembrolizumab in gastric cancer clinical trials, CPS $\geq 10$ was chosen for further evaluation in this analysis to better delineate the specificity of CPS as a predictor of clinical outcomes with pembrolizumab monotherapy. Herein, we characterize clinical outcomes with pembrolizumab monotherapy across lines of therapy in patients with CPS $\geq 10$ advanced gastric or GEJ cancer by analyzing patients with CPS $\geq 10$ tumors enrolled in cohort 1 of KEYNOTE-059 (post hoc analysis), in KEYNOTE-061 (post hoc analysis), and in KEYNOTE-062 (primary analysis).

\section{Patients and Methods \\ Study design}

The designs of KEYNOTE-059 cohort 1, KEYNOTE-061, and KEYNOTE-062 have been described previously (8-10). In brief, all three trials evaluated the efficacy of pembrolizumab $200 \mathrm{mg}$ administered intravenously every 3 weeks for up to 35 cycles ( $\sim 2$ years) for locally advanced unresectable or metastatic gastric or GEJ adenocarcinoma. In KEYNOTE-059, patients were enrolled regardless of PD-L1 expression status. In KEYNOTE-061, patients were randomly assigned 1:1 to receive pembrolizumab monotherapy or standard-dose paclitaxel administered intravenously. Initially, patients were enrolled regardless of PD-L1 expression status, but enrollment was then restricted to those with CPS $\geq 1$ tumors (9). In KEYNOTE-062, patients were randomly assigned (1:1:1) to receive pembrolizumab monotherapy, pembrolizumab plus chemotherapy (standard-dose cisplatin plus 5-FU or capecitabine administered intravenously or orally, respectively), or placebo plus chemotherapy (hereafter referred to as chemotherapy); patients were required to have CPS $\geq 1$ tumors (10). The current analysis of KEYNOTE-062 includes only those patients enrolled in the pembrolizumab monotherapy and chemotherapy groups.

PD-L1 expression was assessed in archival or newly collected tumor samples using PD-L1 IHC 22C3 pharmDx (Agilent Technologies; refs. 8-10) and was measured using CPS [defined as the number of PDL1-staining cells (tumor cells, lymphocytes, macrophages) as a proportion of the total number of tumor cells multiplied by 100; ref. 20]. Samples were not reanalyzed for this analysis. For all three trials, the primary analysis populations were patients with CPS $\geq 1$ tumors. Analysis of outcomes in patients with CPS $\geq 10$ was post hoc for KEYNOTE-059 and KEYNOTE-061 but was part of the prespecified primary analysis for KEYNOTE-062.

The study protocols and all amendments were approved by the institutional review board or ethics committee at each participating institution. The studies were conducted in accordance with the protocol and its amendments and with Good Clinical Practice guidelines. All patients provided written informed consent before enrollment.

\section{Outcomes and statistical considerations}

For the current analysis, we evaluated clinical outcomes in all patients with CPS $\geq 10$ tumors who received $\geq 1$ dose of study drug. Results were analyzed for each of the trials separately (i.e., results were not pooled across trials). Efficacy endpoints included OS, PFS, ORR [complete response (CR) plus partial response (PR)], and DOR.
Response was assessed by central review per RECIST v1.1. The Kaplan-Meier method was used to calculate OS, PFS, and DOR. HRs and their associated 95\% CIs were calculated using stratified Cox proportional hazards models with the Efron method of tie handling. In KEYNOTE-059, ORR was calculated using the Clopper-Pearson method. In KEYNOTE-061 and KEYNOTE-062, treatment differences in OS and PFS were assessed using the log-rank test with HRs estimated using a stratified Cox regression model. Response rate was compared using the Miettinen and Nurminen method. In KEYNOTE062, the prespecified hypotheses included OS analysis of pembrolizumab versus chemotherapy in patients with PD-L1 CPS $\geq 10$ with a planned enrollment for $80 \%$ power to detect a HR of 0.58 at alpha $=$ $0.75 \%$ (one-sided). Full details of the statistical analysis have been published previously (10).

Data cut-off dates for this analysis were August 8, 2018, for KEYNOTE-059, October 26, 2017, for KEYNOTE-061, and March 26, 2019, for KEYNOTE-062.

All three trials are registered with ClinicalTrials.gov [NCT02335411 (KEYNOTE-059), NCT02370498 (KEYNOTE-061), NCT02494583 (KEYNOTE-062)].

\section{Data sharing}

Merck Sharp \& Dohme Corp., a subsidiary of Merck \& Co., Inc., Kenilworth, NJ (MSD) is committed to providing qualified scientific researchers access to anonymized data and clinical study reports from the company's clinical trials for the purpose of conducting legitimate scientific research. MSD is also obligated to protect the rights and privacy of trial participants and, as such, has a procedure in place for evaluating and fulfilling requests for sharing company clinical trial data with qualified external scientific researchers. The MSD datasharing website (available at: http://engagezone.msd.com/ds_docu mentation.php) outlines the process and requirements for submitting a data request. Applications will be promptly assessed for completeness and policy compliance. Feasible requests will be reviewed by a committee of MSD subject matter experts to assess the scientific validity of the request and the qualifications of the requestors. In line with data privacy legislation, submitters of approved requests must enter into a standard data-sharing agreement with MSD before data access is granted. Data will be made available for request after product approval in the United States and European Union or after product development is discontinued. There are circumstances that may prevent MSD from sharing requested data, including country or region-specific regulations. If the request is declined, it will be communicated to the investigator. Access to genetic or exploratory biomarker data requires a detailed, hypothesis-driven statistical analysis plan that is collaboratively developed by the requestor and MSD subject matter experts; after approval of the statistical analysis plan and execution of a datasharing agreement, MSD will either perform the proposed analyses and share the results with the requestor or will construct biomarker covariates and add them to a file with clinical data that is uploaded to an analysis portal so that the requestor can perform the proposed analyses.

\section{Results}

All patients enrolled in KEYNOTE-059, KEYNOTE-061, and KEYNOTE-062 had evaluable tumor samples for PD-L1 status with the exception of 2 patients each in KEYNOTE-059 cohort 1 and KEYNOTE-061; 31\% (46/148), 18\% (108/592), and 36\% (182/506), respectively, had CPS $\geq 10$ tumors (Table 1). Follow-up duration is reported in Table 1. Baseline characteristics for patients with CPS $\geq 10$ tumors 
Wainberg et al.

Table 1. Incidence of PD-L1-positive tumors and follow-up of patients with CPS $\geq 10$ tumors.

\begin{tabular}{|c|c|c|c|c|c|}
\hline \multirow[b]{2}{*}{ Incidence } & \multirow{2}{*}{$\begin{array}{l}\text { KEYNOTE-059 } \\
\text { Pembrolizumab }\end{array}$} & \multicolumn{2}{|c|}{ KEYNOTE-061 } & \multicolumn{2}{|c|}{ KEYNOTE-062 } \\
\hline & & Pembrolizumab & Chemotherapy & Pembrolizumab & Chemotherapy \\
\hline Patients with CPS $\geq 1, n / N(\%)$ & $148 / 259(57)$ & 196/296 (66) & 199/296 (67) & $256 / 256(100)$ & $250 / 250(100)$ \\
\hline Patients with CPS $\geq 10, n / N(\%)$ & $46 / 259(18)$ & $53 / 296(18)$ & $55 / 296(19)$ & $92 / 256(36)$ & $90 / 250(36)$ \\
\hline Median follow-up (range), months & $6(<1-38)$ & $10(<1-28)$ & $8(1-27)$ & $17(<1-38)$ & $11(1-35)$ \\
\hline
\end{tabular}

Abbreviations: CPS, combined positive score; PD-L1, programmed death ligand 1.

were generally comparable between the pembrolizumab and chemotherapy groups in KEYNOTE-061 and KEYNOTE-062 (Table 2).

\section{OS and PFS in the CPS $\geq 10$ population}

In KEYNOTE-059, median OS was 8 months (95\% CI, 5.8-11.1). OS rates were $33 \%$ at 12 months and $15 \%$ at 24 months (Fig. 1A). In KEYNOTE-061, median OS was 10 months (95\% CI, 5.9-17.3) with pembrolizumab and 8 months (95\% CI, 5.1-9.9) with chemotherapy (HR, 0.64; 95\% CI, 0.41-1.02). The OS rates for pembrolizumab and chemotherapy were $45 \%$ versus $23 \%$ at 12 months and $35 \%$ versus $18 \%$ at 18 months, respectively (Fig. 1B). In KEYNOTE-062, median OS was 17 months $(95 \% \mathrm{CI}, 9.1-23.1)$ with pembrolizumab and 11 months (95\% CI, 8.5-13.8) with chemotherapy (HR 0.69; 95\% CI, 0.49-0.97). The OS rates for pembrolizumab and chemotherapy were $57 \%$ versus $47 \%$ at 12 months and $39 \%$ versus $22 \%$ at 24 months, respectively (Fig. 1C). Kaplan-Meier curves showed improved OS in the CPS $\geq 10$ population compared with the CPS $\geq 1$ population from the original studies (Fig. 1A-C).

In KEYNOTE-059, median PFS was 2 months (95\% CI, 2.03.4; Fig. 2A). In KEYNOTE-061, median PFS was 3 months (95\%
CI, 1.4-3.1) with pembrolizumab and 3 months (95\% CI, 2.7-4.1) with chemotherapy (HR, 0.86; 95\% CI, 0.56-1.33; Fig. 2B). In KEYNOTE062, median PFS was 3 months (95\% CI, 1.6-5.4) with pembrolizumab and 6 months (95\% CI, 5.4-6.9) with chemotherapy (HR, 1.09; 95\% CI 0.79-1.49; Fig. 2C). Kaplan-Meier curves of PFS in the CPS $\geq 10$ population compared with the CPS $\geq 1$ population from the original studies are shown in Fig. 2A-C.

\section{Response in the CPS $\geq 10$ population}

In KEYNOTE-059, the confirmed ORR was $17 \%(n=8) ; 1$ patient achieved CR and 7 achieved PR (Table 3). The median DOR was 21 months (range, $3+$ to $35+$; Fig. 3A); five responders (71\%) had a response duration $\geq 6$ months. In KEYNOTE-061, confirmed ORR was $25 \%(n=13)$ for pembrolizumab-treated patients; 5 patients achieved CR and 8 PR (Table 3). In chemotherapy-treated patients, the ORR was $9 \%(n=5) ; 1$ patient achieved CR and 4 achieved PR. The median DOR was not reached (range, 4 to $26+$ months) for pembrolizumab and was 7 months (range, 3-7) for chemotherapy (Fig. 3B); 10 responders $(77 \%)$ treated with pembrolizumab and one responder (53\%) treated with chemotherapy had a response duration $\geq 6$ months.

Table 2. Baseline characteristic of patients with CPS $\geq 10$ tumors.

\begin{tabular}{|c|c|c|c|c|c|}
\hline \multirow[b]{2}{*}{ Characteristic } & \multirow{2}{*}{$\begin{array}{l}\text { KEYNOTE-059 } \\
\text { Pembrolizumab } \\
n=46\end{array}$} & \multicolumn{2}{|c|}{$\begin{array}{l}\text { KEYNOTE-061 } \\
\end{array}$} & \multicolumn{2}{|c|}{$\begin{array}{l}\text { KEYNOTE-062 } \\
\end{array}$} \\
\hline & & $\begin{array}{l}\text { Pembrolizumab } \\
n=53\end{array}$ & $\begin{array}{l}\text { Chemotherapy } \\
n=55\end{array}$ & $\begin{array}{l}\text { Pembrolizumab } \\
n=92\end{array}$ & $\begin{array}{l}\text { Chemotherapy } \\
n=90\end{array}$ \\
\hline Median age, years (range) & $63(30-79)$ & $66(35-79)$ & $60(37-76)$ & $59(20-81)$ & $65(31-82)$ \\
\hline Male, $n(\%)$ & $34(74)$ & $35(66)$ & $35(64)$ & $64(70)$ & $64(71)$ \\
\hline \multicolumn{6}{|l|}{ Race, $n(\%)$} \\
\hline White & $38(83)$ & $34(64)$ & $38(69)$ & $58(63)$ & $58(64)$ \\
\hline Asian & $3(7)$ & $17(32)$ & $13(24)$ & $27(29)$ & $23(26)$ \\
\hline Black & $1(2)$ & $1(2)$ & $1(2)$ & $2(2)$ & $1(1)$ \\
\hline $\begin{array}{l}\text { American Indian or Alaska } \\
\text { Native }\end{array}$ & 0 & $1(2)$ & $2(4)$ & $3(3)$ & $5(6)$ \\
\hline Multiple & $1(2)$ & 0 & $1(2)$ & $1(1)$ & $3(3)$ \\
\hline Missing & $3(7)$ & 0 & 0 & 0 & 0 \\
\hline \multicolumn{6}{|l|}{ ECOG PS, $n(\%)$} \\
\hline 0 & $25(54)$ & $24(45)$ & $24(44)$ & $47(51)$ & $34(38)$ \\
\hline 1 & $21(46)$ & $29(55)$ & $31(56)$ & $45(49)$ & $56(62)$ \\
\hline \multicolumn{6}{|c|}{ No. of previous therapies for metastatic disease, $n$ (\%) } \\
\hline 2 & $21(46)$ & - & - & - & - \\
\hline 3 & $14(30)$ & - & - & - & - \\
\hline 4 & $8(17)$ & - & - & - & - \\
\hline$\geq 5$ & $3(7)$ & - & - & - & - \\
\hline \multicolumn{6}{|l|}{ Tumor site, $n(\%)^{\mathrm{a}}$} \\
\hline Stomach & $22(48)$ & $35(66)$ & $35(64)$ & $68(74)$ & $69(77)$ \\
\hline GEJ & $23(50)$ & $18(34)$ & $20(36)$ & $24(26)$ & $20(22)$ \\
\hline MSI-H, $n$ (\%) & $2(4)$ & $8(15)$ & $5(9)$ & $11(12)$ & $10(11)$ \\
\hline
\end{tabular}

Abbreviations: CPS, combined positive score; ECOG PS, Eastern Cooperative Oncology Group performance status; GEJ, gastroesophageal junction. MSI-H, microsatellite instability-high.

an KEYNOTE-062, one patient (1.1\%) had a tumor site of "missing." 
Efficacy of Pembrolizumab for CPS 10 G/GEJ Cancer

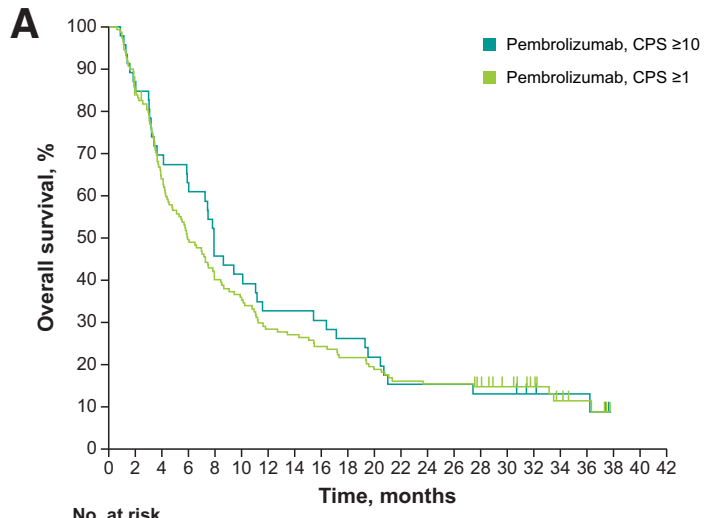

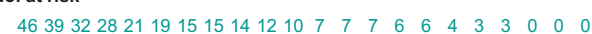
148124937158514139353127232222181510604000
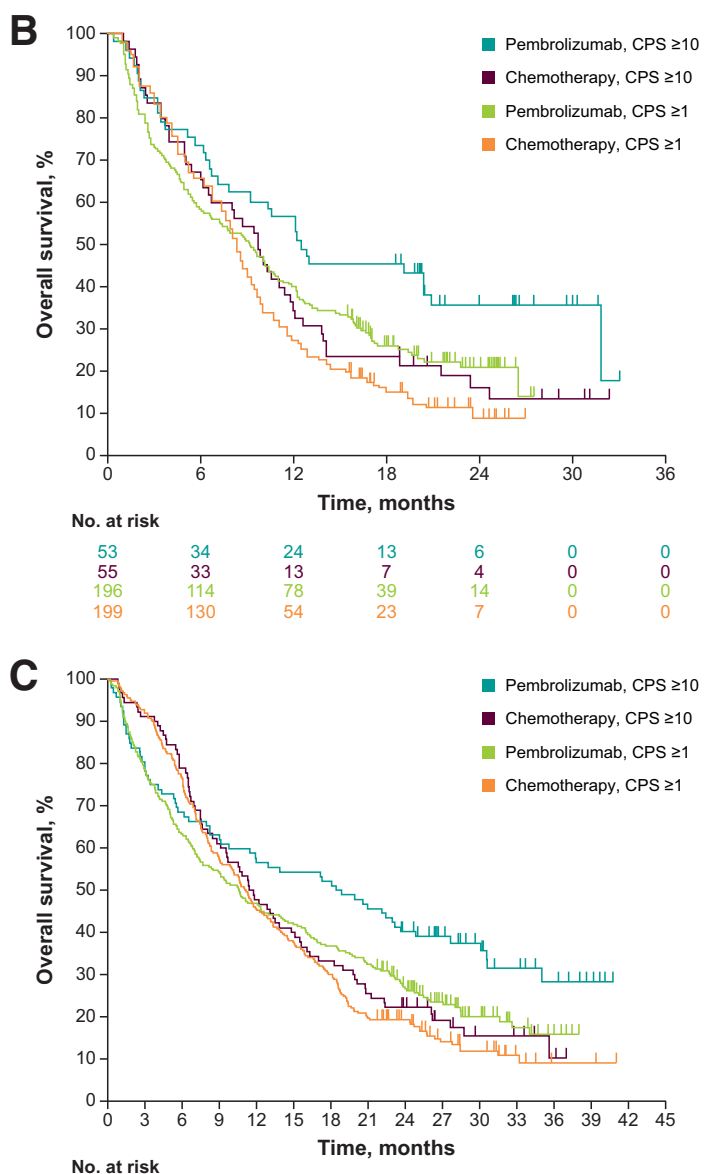
No. at risk

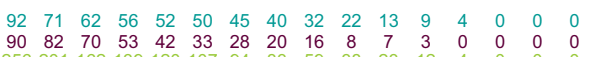

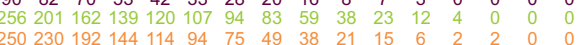

Figure 1.

Kaplan-Meier estimates of OS in patients with CPS $\geq 1$ and CPS $\geq 10$ tumors. A, Patients receiving third-line and later pembrolizumab in KEYNOTE-059 cohort 1. B, Patients receiving second-line pembrolizumab or chemotherapy in KEYNOTE-061. C, Patients receiving first-line pembrolizumab or chemotherapy in KEYNOTE-062. CPS, combined positive score; OS, overall survival.
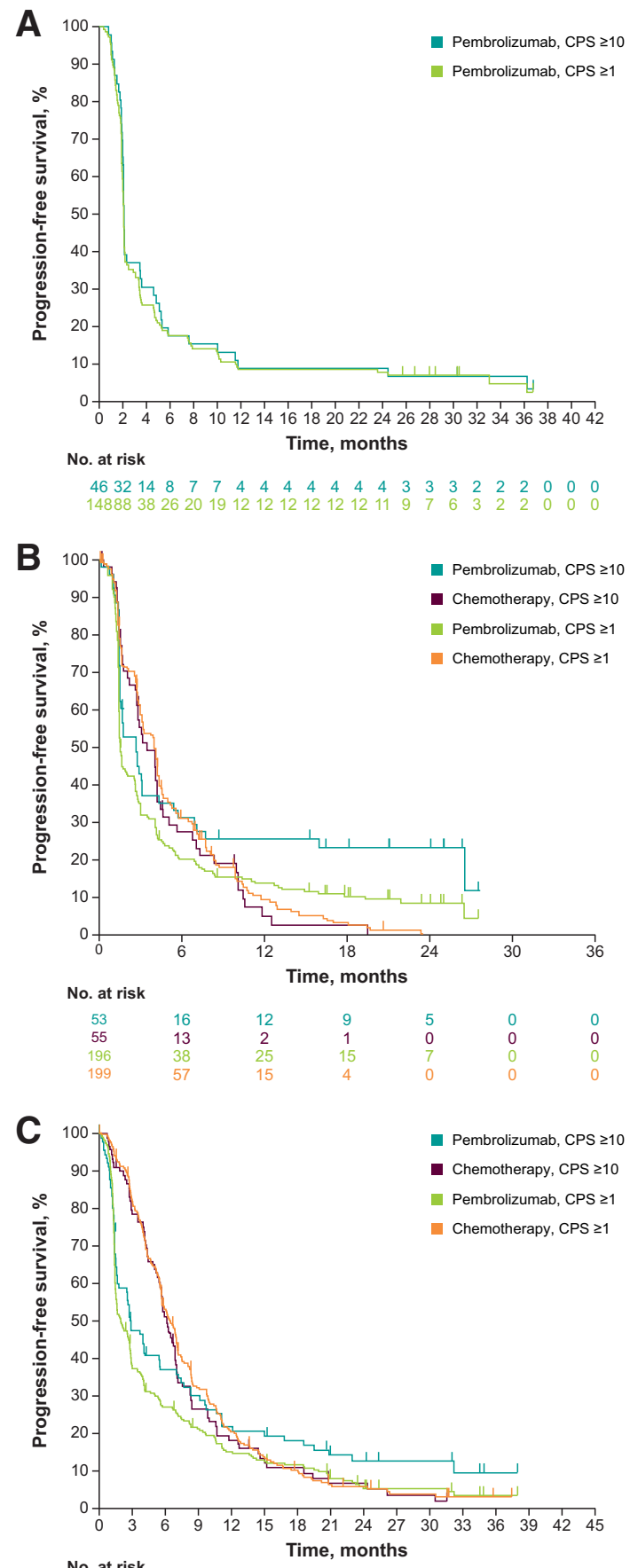

No. at risk Time, months

$\begin{array}{llllllllllllllll}92 & 41 & 31 & 25 & 18 & 17 & 14 & 9 & 8 & 5 & 5 & 3 & 1 & 0 & 0 & 0 \\ 90 & 69 & 44 & 22 & 15 & 10 & 8 & 4 & 4 & 2 & 2 & 0 & 0 & 0 & 0 & 0\end{array}$

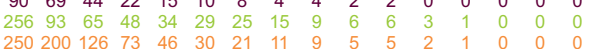

Figure 2.

Kaplan-Meier estimates of PFS in patients with CPS $\geq 1$ and CPS $\geq 10$ tumors. A, Patients receiving third-line and later pembrolizumab in KEYNOTE-059 cohort 1. B, Patients receiving second-line pembrolizumab or chemotherapy in KEYNOTE-061. C, Patients receiving first-line pembrolizumab or chemotherapy in KEYNOTE-062. CPS, combined positive score; PFS, progressionfree survival. 
Table 3. Response summary in patients with CPS $\geq 10$ tumors.

\begin{tabular}{llllll}
\hline & $\begin{array}{l}\text { KEYNOTE-059 } \\
\text { Pembrolizumab }\end{array}$ & \multicolumn{2}{c}{ KEYNOTE-061 } & \multicolumn{2}{c}{ KEYNOTE-062 } \\
& $\boldsymbol{n}=\mathbf{4 6}$ & $\begin{array}{l}\text { Pembrolizumab } \\
\boldsymbol{n}=\mathbf{5 3}\end{array}$ & $\begin{array}{l}\text { Chemotherapy } \\
\boldsymbol{n}=\mathbf{5 5}\end{array}$ & $\begin{array}{l}\text { Pembrolizumab } \\
\boldsymbol{n}=\mathbf{9 2}\end{array}$ & $\begin{array}{l}\text { Chemotherapy } \\
\boldsymbol{n}=\mathbf{9 0}\end{array}$ \\
\hline ORR, $n(\%)$ & $8(17)$ & $13(25)$ & $5(9)$ & $23(25)$ & $34(38)$ \\
CR & $1(2)$ & $5(9)$ & $1(2)$ & $7(8)$ & $4(4)$ \\
PR & $7(15)$ & $8(15)$ & $4(7)$ & $16(17)$ & $30(33)$ \\
SD & $9(20)$ & $12(23)$ & $28(51)$ & $23(25)$ & $39(43)$ \\
PD & $24(52)$ & $23(43)$ & $11(20)$ & $29(32)$ & $8(9)$ \\
$\quad$ Not available & $5(11)$ & $5(9)$ & $11(20)$ & $17(19)$ & $9(10)$ \\
Median time to response, months, & $2(2-4)$ & $2(1-3)$ & $2(1-4)$ & $1(1-7)$ & $2(1-7)$ \\
$\quad$ (range) & $21(3+$ to 35+) & NR (4 to 26+) & $7(3$ to 7) & $19(1+$ to 34+) & $7(2+$ to 30+) \\
Median DOR, months, (range) & &
\end{tabular}

Abbreviations: $\mathrm{Cl}$, confidence interval; $\mathrm{CPS}$, combined positive score; $\mathrm{CR}$, complete response; $\mathrm{DOR}$, duration of response; NR, not reached; PD, progressive disease; $\mathrm{PR}$, partial response; SD, stable disease.

andicates patients without an evaluable assessment or patients who had a baseline assessment but no postbaseline assessment as of the data cut-off date (due to missing, discontinuing, or death before the first postbaseline assessment).

In KEYNOTE-062, confirmed ORR was $25 \%(n=23)$ for pembrolizumab-treated patients; 7 patients achieved CR and 16 achieved PR (Table 3). In chemotherapy-treated patients, the ORR was $38 \%(n=$ 34); 4 patients achieved CR and 30 achieved PR. The median DOR was 19 months (range, $1+$ to $34+$ ) for pembrolizumab and 7 months (range, $2+$ to $30+$ ) for chemotherapy (Fig. 3C); 18 responders (82\%) treated with pembrolizumab and 16 responders (53\%) treated with chemotherapy had a response duration $\geq 6$ months. Kaplan-Meier curves showed DOR in the CPS $\geq 10$ population compared with the CPS $\geq 1$ population from the original studies.

\section{Discussion}

In the primary analysis of patients with CPS $\geq 1$ gastric or GEJ cancer who were enrolled in KEYNOTE-059 cohort 1, KEYNOTE061, and KEYNOTE-062, pembrolizumab monotherapy demonstrated promising antitumor activity. In KEYNOTE-061 and KEYNOTE062 , pembrolizumab was associated with an improved safety profile, but it did not significantly improve survival outcomes compared with chemotherapy (8-10). The current analysis in patients with CPS $\geq 10$ tumors revealed durable responses and elongation of the tails of the Kaplan-Meier OS curves with pembrolizumab monotherapy across lines of therapy. However, pembrolizumab monotherapy did not numerically improve PFS in this analysis of KEYNOTE-061 or KEYNOTE-062 or ORR in KEYNOTE-062 compared with chemotherapy. The relationship between OS and PFS in clinical trials of immune checkpoint inhibitors has been investigated in several tumor types, including gastric cancer; differences in PFS and OS benefit as well as direction of outcomes are likely attributable to the mechanism of action, specific disease, and population under study (21).

In addition to other factors including MSI and HER2 status, PD-L1 expression can provide important guidance for patient selection in clinical practice and is used to select patients eligible for pembrolizumab therapy. On the basis of a recent meta-analysis showing an expression-response relationship between PD-L1 and OS, we evaluated whether an increase in PD-L1 positivity from CPS $\geq 1$ to CPS $\geq 10$ resulted in improved responses to pembrolizumab (19). In comparing the current analysis of CPS $\geq 10$ tumors, in which patient numbers are small, with previously reported data in patients with CPS $\geq 1$ tumors, we observed numerically higher median OS, ORR, and DOR with pembrolizumab therapy by increasing the CPS cutoff from $\geq 1$ to $\geq 10$. In KEYNOTE-059, median OS increased from 6 to 8 months, and the 12-month OS rate increased from $24 \%$ to $33 \%$, the ORR increased from $16 \%$ to $17 \%$, and the DOR increased from 16 to 21 months (8). In KEYNOTE-061, median OS increased from 9 to 10 months, and the 12 -month OS rate increased from $40 \%$ to $45 \%$, the 18 -month OS rate increased from $26 \%$ to $35 \%$, the ORR increased from $16 \%$ to $25 \%$, and the DOR increased from 18 months to not reached $(9,22)$. In KEYNOTE-062, median OS increased from 11 to 17 months, and the 12 -month OS rate increased from $47 \%$ to $57 \%$, the 24 -month OS rate increased from $27 \%$ to $39 \%$, the ORR increased from $15 \%$ to $25 \%$, and the DOR increased from 14 to 19 months (10). In KEYNOTE-061, the HR for OS decreased from 0.82 for CPS $\geq 1$ to 0.64 for CPS $\geq 10$ (9), and in KEYNOTE-062, the HR for OS decreased from 0.91 for CPS $\geq 1$ to 0.69 for CPS $\geq 10$ (10). In KEYNOTE-062, the combination of pembrolizumab and chemotherapy was not superior to chemotherapy for OS in patients with CPS $\geq 1$ or CPS $\geq 10$ tumors (10). Thus, increasing the CPS cutoff to CPS $\geq 10$ in patients with gastric or GEJ cancer may provide greater treatment benefit for patients eligible to receive pembrolizumab monotherapy.

The clinical benefit of using higher PD-L1 cutoffs with pembrolizumab has also been evaluated in other tumor types. Evidence from the phase III KEYNOTE-181 study in patients with advanced/metastatic esophageal cancer demonstrated a significant benefit with a high CPS cutoff. Among 222 patients with CPS $\geq 10$ tumors, second-line pembrolizumab monotherapy significantly improved OS versus chemotherapy (HR, 0.69; 95\% CI, 0.52-0.93; $P=0.0074$; ref. 23). In the phase III KEYNOTE-048 trial in patients with untreated locally incurable recurrent or metastatic head and neck squamous cell carcinoma, pembrolizumab monotherapy demonstrated a greater survival benefit than cetuximab plus chemotherapy in the population with CPS $\geq 20$ tumors (HR, 0.61 ; 95\% CI, 0.45$0.83 ; P=0.0007)$ than in the population with CPS $\geq 1$ tumors (HR, 0.78 ; $95 \% \mathrm{CI}, 0.64-0.96$; $P=0.0086$; ref. 24 ). In the single-arm phase II KEYNOTE-052 study in patients with locally advanced and unresectable or metastatic urothelial cancer, response to pembrolizumab monotherapy increased with increasing CPS cutoff (CPS $\geq$ $1,11 \%$; CPS $>1$ to $<10,20 \%$; CPS $\geq 10,39 \%$; ref. 25 ). In patients with advanced recurrent ovarian cancer enrolled in the phase II KEYNOTE-100 study, higher PD-L1 expression also correlated with higher response to pembrolizumab monotherapy (CPS $\geq 1$, $5.7 \%$; CPS $\geq 10,10.0 \%$; ref. 26 ).

Limitations of the current analysis include the post hoc nature of KEYNOTE-059 cohort 1 and KEYNOTE-061 and the small patient 
Efficacy of Pembrolizumab for CPS 10 G/GEJ Cancer
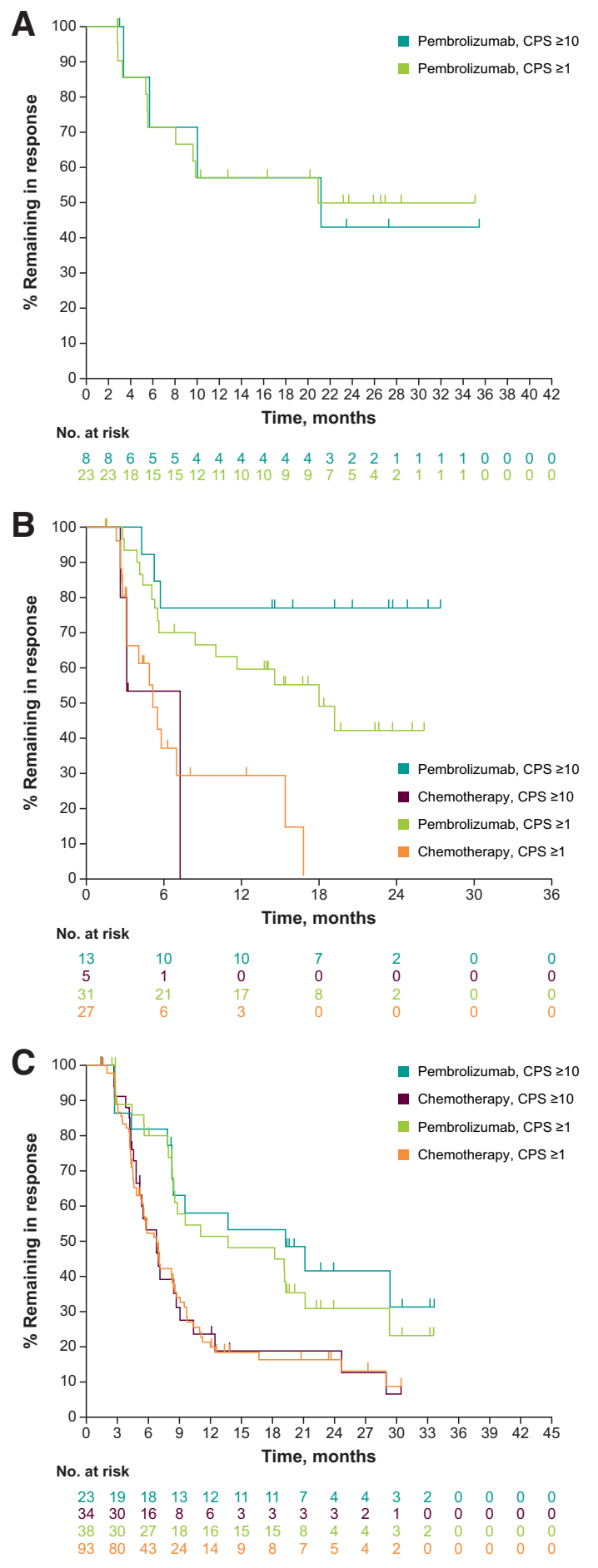

Figure 3.

Kaplan-Meier estimates of DOR in patients with CPS $\geq 1$ and CPS $\geq 10$ tumors. A, Patients receiving third-line and later pembrolizumab in KEYNOTE-059 cohort 1. B, Patients receiving second-line pembrolizumab or chemotherapy in KEYNOTE-061. C, Patients receiving first-line pembrolizumab or chemotherapy in KEYNOTE-062. CPS, combined positive score; DOR, duration of response. numbers within each subgroup. Furthermore, biomarker enrichment can predict response, but prevalence can decrease with higher CPS enrichment. Taken together, definitive conclusions cannot be made from this analysis.

In this analysis, these data suggest that pembrolizumab monotherapy given as first-line (KEYNOTE-062), second-line (KEYNOTE061), and third-line and later (KEYNOTE-059) therapy showed a clinically meaningful median and long-term survival benefit in patients with CPS $\geq 10$ gastric or GEJ tumors and more durable responses compared with chemotherapy. This study adds to the existing body of evidence that the immunohistochemical PD-L1 CPS is one clinically relevant biomarker that can lead to improved clinical efficacy and validates the importance of refining the PD-L1 CPS biomarker companion diagnostic as we attempt to define the optimal role of pembrolizumab in gastric cancer. Although evidence from the current analysis and in other tumor types has validated scoring of PDL1 expression using tumor and immune cells (i.e., CPS) to predict response to pembrolizumab, large and prospective trials are needed to validate the optimal CPS cutoff for patients with gastric or GEJ cancer.

\section{Authors' Disclosures}

Z.A. Wainberg reports advisory/consultancy for AstraZeneca, Bayer, Daiichi, FivePrime, Lilly, Merck, and Molecular Therapeutics; research grant/funding (to his institution) from AstraZeneca, Daiichi, FivePrime, Lilly, and Merck; and travel/ accommodations/expenses for Bayer, Daiichi, Lilly, Merck, and Molecular Therapeutics. C.S. Fuchs reports consulting for Agios, Amylin Pharmaceuticals, Bain Capital, CytomX Therapeutics, Daiichi-Sankyo, Eli Lilly, Entrinsic Health, Evolveimmune Therapeutics, Genentech, Merck, Taiho, and Unum Therapeutics; serves as a director for CytomX Therapeutics and owns unexercised stock options for CytomX and Entrinsic Health; is a co-founder of EvolveImmune Therapeutics and has equity in this private company; and has provided expert testimony for Amylin Pharmaceuticals and Eli Lilly. J. Tabernero reports consultancy for Array Biopharma, AstraZeneca, Bayer, BeiGene, Biocartis, Boehringer-Ingelheim, Chugai, F. Hoffmann-La Roche Ltd, Foundation Medicine, Genentech Inc, Genmab A/S, HalioDX SAS, Halozyme, Imugene Limited, Inflection Biosciences Limited, Ipsen, Kura Oncology, Lilly, MSD, Menarini, Merck Serono, Merrimack, Merus, Molecular Partners, Novartis, Peptomyc, Pfizer, Pharmacyclics, ProteoDesign SL, Rafael Pharmaceuticals, Roche Diagnostics, Sanofi, SeaGen, Seattle Genetics, Servier, Symphogen, Taiho, and VCN Biosciences. K. Shitara reports an advisory role for AbbVie Inc, Astellas Pharma, Bristol-Myers Squibb, Eli Lilly and Company, GlaxoSmithKline, Merck, Novartis, Ono Pharmaceutical, Pfizer Inc, Taiho Pharmaceutical, and Takeda Pharmaceuticals; research funding for Astella Pharma, Chugai Pharma, Dainippon Sumitomo Pharma, Daiichi Sankyo, Eli Lilly and Company, Medi Science, Merck, Ono Pharmaceutical, and Taiho Pharmaceutical; and honoraria (lecture fee) for AbbVie Inc, Novartis, and Yakult. K. Muro reports research funding (to his institution) from Daiichi Sankyo, Mediscience Planning, MSD, Parexel International, Pfizer, Sanofi, Solasia Pharma, and Sumitomo Dainippon Pharma; honoraria for speaking from Bristol-Myers Squibb, Chugai Pharmaceutical, Eli Lilly, Ono Pharmaceutical Co., Ltd., Taiho Pharmaceutical, Takeda Pharmaceutical, and Sanofi; and advisory/consultancy for Amgen, AstraZeneca, and Ono Pharmaceutical Co., Ltd. E. Van Cutsem reports consulting/advisory role for Array, AstraZeneca, Bayer, Biocartis, Bristol-Myers Squibb, Celgene, Daiichi, Halozyme, GSK, Pierre-Fabre, Incyte, Ipsen, Lilly, Merck Sharp \& Dohme Corp., a subsidiary of Merck \& Co., Inc., Kenilworth, NJ, Merck KGaA, Novartis, Pierre Fabre, Roche, Servier, Sirtex, and Taiho; and grants (to institution) from Amgen, Bayer, Boehringer Ingelheim, BristolMyers Squibb, Celgene, Ipsen, Lilly, Merck Sharp \& Dohme Corp., a subsidiary of Merck \& Co., Inc., Kenilworth, NJ, Merck KGaA, Novartis, Roche, and Servier. Y.-J. Bang reports consulting/advisory for Astellas, AstraZeneca, Bayer, BeiGene, BMS, Daiichi-Sankyo, Eli Lilly, Genentech/Roche, Genexine, GreenCross, Hammi, MerckSerono, MSD, Novartis, Samyang Biopharm, and Taiho, and grants (to the institution for clinical trials) from Astellas, AstraZeneca, Bayer, BeiGene, Boehringer-Ingelheim, Bostin Biomedical, BMS, CKD Pharma, Curis, Daiichi-Sanyko, Eli Lilly, FivePrime, Genentech/Roche, Genexine GreenCross, GSK, MacroGenics, Merck Serono, MSD, Novartis, Ono, Pfizer, Taiho, and Takeda. H.C. Chung reports research support from Amgen, BeiGene, BMS/Ono, GSK, Lilly, MSD, Merck-Serono, and Taiho; honoraria from Lilly and Merck-Serono; and consultancy for Amgen, BeiGene, BMS, Celltrion, Gloria, Lilly, Merck-Serono, MSD, Quintiles, Taiho, and Zymework. K. Yamaguchi 
reports consulting/advisory for Bristol-Myers Squibb and Daiichi Sankyo; speakers' bureau for Bristol-Myers Squibb, Chugai Pharma, Daiichi Sankyo, Lilly, Merck Serono, Ono Pharmaceutical, Sanofi, Taiho Pharmaceutical, and Takeda: and research funding (institution) from Boehringer Ingelheim, Chugai Pharma, Daiichi Sankyo, Eisai, Gilead Sciences, Lilly, MSD Oncology, Ono Pharmaceutical, Sumitomo Dainippon Pharma, Taiho Pharmaceutical, and Yakult Honsha D. Hochhauser reports stock or other ownership in Novartis and Roche; research funding from Merck Serono; and travel, accommodations, and expenses from Merck P. Thuss-Patience reports advisory board for BMS, Lilly, Merck Serono, MSD, Pfizer, Roche, and Servier; and study support from Merck Serono. U. Kher is an employee of Merck Sharp \& Dohme Corp., a subsidiary of Merck \& Co., Inc., Kenilworth, NJ. C.-S. Shih is an employee of and a stockholder in Merck. No disclosures were reported by the other authors.

\section{Authors' Contributions}

Z.A. Wainberg: Conceptualization, resources, data curation, formal analysis, writing-original draft, writing-review and editing. C.S. Fuchs: Conceptualization, resources, data curation, writing-review and editing. J. Tabernero: Conceptualization, resources, data curation, formal analysis, writing-review and editing. K. Shitara: Conceptualization, resources, data curation, writing-review and editing. K. Muro: Resources, writing-review and editing. E. Van Cutsem: Conceptualization, resources, data curation, writing-review and editing. Y.-J. Bang: Resources, data curation, writing-review and editing. H.C. Chung: Resources, data curation, writingoriginal draft, writing-review and editing. K. Yamaguchi: Data curation, writing-

\section{References}

1. Bray F, Ferlay J, Soerjomataram I, Siegel RL, Torre LA, Jemal A. Global cancer statistics 2018: GLOBOCAN estimates of incidence and mortality worldwide for 36 cancers in 185 countries. CA Cancer J Clin 2018;68: 394-424.

2. Zhang M, Dong Y, Liu H, Wang Y, Zhao S, Xuan Q, et al. The clinicopathological and prognostic significance of PD-L1 expression in gastric cancer: a metaanalysis of 10 studies with 1,901 patients. Sci Rep 2016;6:37933.

3. Gu L, Chen M, Guo D, Zhu H, Zhang W, Pan J, et al. PD-L1 and gastric cancer prognosis: a systematic review and meta-analysis. PLoS One 2017;12: e0182692.

4. Liu YX, Wang XS, Wang YF, Hu XC, Yan JQ, Zhang YL, et al. Prognostic significance of PD-L1 expression in patients with gastric cancer in East Asia: a meta-analysis. OncoTargets Ther 2016;9:2649-54.

5. Kawazoe A, Kuwata T, Kuboki Y, Shitara K, Nagatsuma AK, Aizawa M, et al. Clinicopathological features of programmed death ligand 1 expression with tumor-infiltrating lymphocyte, mismatch repair, and Epstein-Barr virus status in a large cohort of gastric cancer patients. Gastric Cancer 2017;20:407-15.

6. National Comprehensive Cancer Network. NCCN clinical practice guidelines in oncology: gastric cancer (Version 3.2020); 2020. Available from: https://www. nccn.org/professionals/physician_gls/pdf/gastric.pdf.

7. Fashoyin-Aje L, Donoghue M, Chen H, He K, Veeraraghavan J, Goldberg KB, et al. FDA approval summary: pembrolizumab for recurrent locally advanced or metastatic gastric or gastroesophageal junction adenocarcinoma expressing PDL1. Oncologist 2019;24:103-9.

8. Fuchs CS, Doi T, Jang RW, Muro K, Satoh T, Machado M, et al. Safety and efficacy of pembrolizumab monotherapy in patients with previously treated advanced gastric and gastroesophageal junction cancer: phase 2 clinical KEYNOTE-059 trial. JAMA Oncol 2018;4:e180013.

9. Shitara K, Ozguroglu M, Bang YJ, Di Bartolomeo M, Mandala M, Ryu MH, et al. Pembrolizumab versus paclitaxel for previously treated, advanced gastric or gastro-oesophageal junction cancer (KEYNOTE-061): a randomised, openlabel, controlled, phase 3 trial. Lancet 2018;392:123-33.

10. Shitara K, Van Cutsem E, Bang YJ, Fuchs CS, Wyrwicz L, Lee KW, et al. Efficacy and safety of pembrolizumab or pembrolizumab plus chemotherapy vs chemotherapy alone for patients with first-line, advanced gastric cancer: the KEYNOTE-062 phase 3 randomized clinical trial. JAMA Oncol 2020;6:1571-80.

11. Janjigian YY, Bendell J, Calvo E, Kim JW, Ascierto PA, Sharma P, et al. CheckMate-032 study: efficacy and safety of nivolumab and nivolumab plus ipilimumab in patients with metastatic esophagogastric cancer. J Clin Oncol 2018;36:2836-44. original draft, writing-review and editing. E. Varga: Conceptualization, resources, data curation, formal analysis, writing-original draft, writing-review and editing. J.-S. Chen: Resources, data curation, writing-review and editing. D. Hochhauser: Resources, writing-review and editing. P. Thuss-Patience: Resources, data curation, writingreview and editing. S.-E. Al-Batran: Resources, writing-review and editing. M. Garrido: Resources, data curation, formal analysis, writing-review and editing. U. Kher: Data curation, writing-review and editing. C.-S. Shih: Resources, data curation, writing-review and editing. S. Shah: Conceptualization, data curation, writing-review and editing. P. Bhagia: Formal analysis, writing-review and editing. J. Chao: Data curation, writing-review and editing.

\section{Acknowledgments}

The authors thank the patients and their families and all investigators and site personnel. The authors also thank Eric Rubin of Merck Sharp \& Dohme Corp., a subsidiary of Merck \& Co., Inc., Kenilworth, NJ. Medical writing and/or editorial assistance was provided by Holly C. Cappelli, PhD, CMPP, and Brian Szente, PhD, of ApotheCom (Yardley, PA). This assistance was funded by Merck Sharp \& Dohme Corp., a subsidiary of Merck \& Co., Inc., Kenilworth, NJ.

The costs of publication of this article were defrayed in part by the payment of page charges. This article must therefore be hereby marked advertisement in accordance with 18 U.S.C. Section 1734 solely to indicate this fact.

Received July 29, 2020; revised October 16, 2020; accepted January 8, 2021; published first January 14, 2021.

12. Kang YK, Boku N, Satoh T, Ryu MH, Chao Y, Kato K, et al. Nivolumab in patients with advanced gastric or gastro-oesophageal junction cancer refractory to, or intolerant of, at least two previous chemotherapy regimens (ONO-453812, ATTRACTION-2): a randomised, double-blind, placebo-controlled, phase 3 trial. Lancet 2017;390:2461-71.

13. Chung HC, Arkenau HT, Lee J, Rha SY, Oh DY, Wyrwicz L, et al. Avelumab (anti-PD-L1) as first-line switch-maintenance or second-line therapy in patients with advanced gastric or gastroesophageal junction cancer: phase $1 \mathrm{~b}$ results from the JAVELIN Solid Tumor trial. J Immunother Cancer 2019;7:30.

14. Bang YJ, Ruiz EY, Van Cutsem E, Lee KW, Wyrwicz L, Schenker M, et al. Phase III, randomised trial of avelumab versus physician's choice of chemotherapy as third-line treatment of patients with advanced gastric or gastro-oesophageal junction cancer: primary analysis of JAVELIN Gastric 300. Ann Oncol 2018;29: 2052-60.

15. Moehler MH, Dvorkin M, Ozguroglu M, Ryu MH, Muntean AS, Lonardi S, et al. Results of the JAVELIN Gastric 100 phase 3 trial: avelumab maintenance following first-line (1L) chemotherapy (CTx) vs continuation of CTx for HER2 advanced gastric or gastroesophageal junction cancer (GC/GEJC). J Clin Oncol 38: 4s, 2020 (suppl; abstr 278).

16. Helwick C. No survival benefit for maintenance avelumab in advanced gastric or gastroesophageal junction cancer; 2020. Available from: https://www.ascopost. com/issues/march-10-2020/no-survival-benefit-for-maintenance-avelumabin-advanced-gastric-or-gastroesophageal-junction-cancer/.

17. Muro K, Chung HC, Shankaran V, Geva R, Catenacci D, Gupta S, et al. Pembrolizumab for patients with PD-L1-positive advanced gastric cancer (KEYNOTE-012): a multicentre, open-label, phase $1 \mathrm{~b}$ trial. Lancet Oncol 2016;17:717-26.

18. Moehler M, Shitara K, Garrido M, Salman P, Shen L, Wyrwicz L, et al. Nivolumab (nivo) plus chemotherapy (chemo) versus chemo as first-line (1L) treatment for advanced gastric cancer/gastroesophageal junction cancer (GC/GEJC)/esophageal adenocarcinoma (EAC): first results of the CheckMate 649 study. Ann Oncol 2020;31:S1191.

19. Liu X, Guo CY, Tou FF, Wen XM, Kuang YK, Zhu Q, et al. Association of PD-L1 expression status with the efficacy of PD-1/PD-L1 inhibitors and overall survival in solid tumours: a systematic review and meta-analysis. Int J Cancer 2020;147: 116-27.

20. Kulangara K, Zhang N, Corigliano E, Guerrero L, Waldroup S, Jaiswal D, et al. Clinical utility of the combined positive score for programmed death ligand-1 expression and the approval of pembrolizumab for treatment of gastric cancer Arch Pathol Lab Med 2019;143:330-7. 
Efficacy of Pembrolizumab for CPS 10 G/GEJ Cancer

21. Hess LM, Brnabic A, Mason O, Lee P, Barker S.. Relationship between progression-free survival and overall survival in randomized clinical trials of targeted and biologic agents in oncology. J Cancer 2019;10:3717-27.

22. Fuchs CS, Ozguroglu M, Bang YJ, Di Bartolomeo M, Mandala M, Ryu MH, et al Pembrolizumab (pembro) vs paclitaxel (PTX) for previously treated advanced gastric or gastroesophageal junction (G/GEJ) cancer: phase 3 KEYNOTE-061 trial. J Clin Oncol 36: 15s, 2018 (suppl; abstr 4062).

23. Kojima T, Shah MA, Muro K, Francois E, Adenis A, Hsu CH, et al. Randomized phase III KEYNOTE-181 study of pembrolizumab versus chemotherapy in advanced esophageal cancer. J Clin Oncol 2020;38:4138-48.

24. Burtness B, Harrington KJ, Greil R, Soulières D, Tahara M, de Castro G, et al Pembrolizumab alone or with chemotherapy versus cetuximab with chemo- therapy for recurrent or metastatic squamous cell carcinoma of the head and neck (KEYNOTE-048): a randomised, open-label, phase 3 study. Lancet 2019; 394:1915-28.

25. Balar AV, Castellano D, O’Donnell PH, Vuky J, Powles T, et al. Firstline pembrolizumab in cisplatin-ineligible patients with locally advanced and unresectable or metastatic urothelial cancer (KEYNOTE-052): a multicentre, single-arm, phase 2 study. Lancet Oncol 2017;18: 1483-92.

26. Matulonis UA, Shapira-Frommer R, Santin AD, Lisyanskaya AS, Pignata S, Vergote I, et al. Antitumor activity and safety of pembrolizumab in patients with advanced recurrent ovarian cancer: results from the phase II KEYNOTE-100 study. Ann Oncol 2019;30:1080-7. 


\section{Clinical Cancer Research}

Efficacy of Pembrolizumab Monotherapy for Advanced Gastric/Gastroesophageal Junction Cancer with Programmed Death Ligand 1 Combined Positive Score $\geq 10$

Zev A. Wainberg, Charles S. Fuchs, Josep Tabernero, et al.

Clin Cancer Res 2021;27:1923-1931. Published OnlineFirst January 14, 2021.

Updated version Access the most recent version of this article at:

doi:10.1158/1078-0432.CCR-20-2980

Cited articles

This article cites 22 articles, 2 of which you can access for free at:

http://clincancerres.aacrjournals.org/content/27/7/1923.full\#ref-list-1

E-mail alerts

Reprints and

Subscriptions

Permissions
Sign up to receive free email-alerts related to this article or journal.

To order reprints of this article or to subscribe to the journal, contact the AACR Publications Department at pubs@aacr.org.

To request permission to re-use all or part of this article, use this link http://clincancerres.aacrjournals.org/content/27/7/1923.

Click on "Request Permissions" which will take you to the Copyright Clearance Center's (CCC)

Rightslink site. 